ORIGINAL ARTICLE

\title{
Size, shape, structure, and direction of angiogenesis in laryngeal tumour development
}

\author{
J Laitakari, V Näyhä, F Stenbäck
}

J Clin Pathol 2004;57:394-401. doi: 10.1136/icp.2002.004978

See end of article for authors' affiliations ....................

Correspondence to: Dr F Stenback, Professor, Department of Pathology, University of Oulu, PO Box 5000, Oulu 90014 Finland; frej.stenback@ oulu.fi

Accepted for publication 19 November 2003

\begin{abstract}
Aims: Angiogenesis and vessel organisation in laryngeal tumour development and progression were examined to determine characteristics of biological and clinical relevance.

Methods: Automated quantitative image analysis was performed on 1451 factor VIII (FVIII) associated blood vessels with regard to occurrence, structure, size, shape, and staining intensity, in addition to vessel direction.

Results: Vessel numbers were increased in preneoplastic states and severe dysplasia, in addition to squamous cell carcinomas, being greater in poorly differentiated carcinomas. Small regular vessels predominated in benign conditions and large, irregular vessels in malignant neoplasms. Vessel distribution was related to degree of differentiation in squamous cell carcinomas, with circumferential angiogenesis occurring in well differentiated neoplasms, directional angiogenesis in moderately differentiated tumours, and aberrant angiogenesis in less well differentiated neoplasms. Alterations in vessel shape increased significantly with increasing degree of malignancy. Comparing the characteristics of individual vessels showed vessel shape abnormalities and the intensity of FVIII staining to increase with vessel size.

Conclusions: Increased angiogenesis was an early event in laryngeal tumour development, with vessel structure, size, and shape related to the tumour growth pattern and behaviour.
\end{abstract}

$\mathrm{T}$ he management of head and neck tumours depends upon clinical findings, histopathological classification, and the TNM stage; tumour size, tumour location, and uninvolved surgical margins also have prognostic significance. ${ }^{1-7}$ Recently developed neoplastic prognostic indicators include p53 expression, c-erb-B2 expression, ${ }^{7}$ and extent of angiogenesis. $^{8-10}$ In head and neck tumours, increased angiogenesis has been associated with an unfavourable prognosis in many studies, ${ }^{8-15}$ whereas others report no associations, ${ }^{16-24}$ and one study reported that decreased angiogenesis was associated with a poor response to treatment. ${ }^{25}$ The prognostic relevance of angiogenic factors in laryngeal tumour development has been questioned, ${ }^{8-10} 12-1526$ and conflicting results have been reported. ${ }^{11}{ }^{16-24}$

"Automated quantitative image analysis allows the accurate study of large numbers of specimens, with a reproducibility and sensitivity exceeding $99 \% "$

A major difficulty in studying angiogenesis in humans is the lack of direct methods for measuring angiogenic activity. A commonly used method is to measure the density of the microvasculature in histological tumour sections. ${ }^{7-9}$ Previous studies assessing angiogenesis have mainly involved histological techniques, factor VIII (FVIII) expression, ${ }^{11} 1927$ and CD31 expression. ${ }^{21}{ }^{28}$ The advent of morphometric methods has provided new possibilities in such studies. ${ }^{89} 2930$ Automated quantitative image analysis allows the accurate study of large numbers of specimens, with a reproducibility and sensitivity exceeding $99 \% .^{30}$ This method allows an exact measurement of cell and tissue size, shape, and organisation, which is not possible with other methods. Using morphometric techniques, we attempted to decrease human error, increase efficiency, assess a large area of tumour sample, create reproducible results, and help to standardise the measurement of angiogenesis..$^{29-31}$
Our study presents results of automated quantitative image analysis of vascular structures in tumour development in the larynx. We report the location and total volume, in addition to size, shape, and staining intensity of FVIII stained vessels in squamous cell carcinomas with different degrees of malignancy and mode of spread, in relation to neoplasm development and behaviour. The purpose was to identify vascular features that may aid in defining the characteristics of laryngeal tumours and possibly identify those patients who would benefit from antiangiogenic treatments.

\section{MATERIALS AND METHODS \\ Material}

We investigated laryngeal specimens from 16 patients, removed at surgery for clinical purposes and studied at the department of pathology, University of Oulu Hospital, Finland. Specimens were removed according to standard clinical procedures and representative areas, 60 altogether, selected for our study. The specimens were all from resections for clinically diagnosed premalignant or malignant conditions. The only criteria for inclusion were sufficient material and histopathologically defined morphology. Histopathological classification was based on epithelial morphology. ${ }^{32}$

For morphometrical analysis, the stroma adjacent to the epithelial tissue, next to the basement membrane (BM), was analysed (10-15 microscope fields of each lesion type; magnification, $\times 100 ; 320 \mu \mathrm{m} \times 600 \mu \mathrm{m}$, in a continuous fashion). Only appropriate areas fulfilling the morphological criteria were analysed and no other selection was done. Almost all vascular structures in the surrounding stroma were analysed and no selection was performed. From the

Abbreviations: $\mathrm{BM}$, basement membrane; $\mathrm{ECM}$, extracellular membrane; FVIII, factor VIII; PBS, phosphate buffered saline 
same specimen, morphologically different lesions showing no relation were analysed separately. Variation between blocks and areas of large sections occurred; vascular structures were classified according to the structure of the associated epithelial lesion. Variation is reflected in the statistical analysis and the confidence intervals presented in the results section. Because of variations in the specimens from the patients prognostic significance was not determined and vessel counts were not adjusted for the amount of tumour in each field.

Formalin fixed, paraffin wax embedded, $5 \mu \mathrm{m}$ thick sections were stained routinely with haematoxylin and eosin, and other stains as appropriate. Table l summarises the numbers of the different types of lesion-normal, mild dysplasia, severe dysplasia, well differentiated (grade I) squamous cell carcinoma, moderately differentiated (grade II) squamous cell carcinoma, and poorly differentiated (grade III) squamous cell carcinoma-and the numbers of vessels.

\section{Immunohistochemical methods}

Rabbit antibodies to human FVIII were purchased from Dako Immunoglobulin A/S (Copenhagen, Denmark) and used according to their specifications. The avidin-biotin modification of the peroxidase-antiperoxidase method was used for the immunohistochemical studies of histological sections. Sections ( $5 \mu \mathrm{m}$ thick) were cut, placed on slides coated with 3-triethoxysilylpropylamine (Sigma, St Louis, Missouri, USA), and fixed at $37^{\circ} \mathrm{C}$ overnight. Slides were dewaxed in graded series of xylene and ethanol. Endogenous peroxidase activity was quenched in $3 \% \mathrm{H}_{2} \mathrm{O}_{2}$ methanol solution for 30 minutes. Slides were then placed in $\mathrm{H}_{2} \mathrm{O}$ and phosphate buffered saline (PBS), and then submitted to pretreatment using $0.4 \%$ pepsin (Merck, Darmstadt, Germany) at $37^{\circ} \mathrm{C}$ for 30 minutes. The slides were subsequently rinsed with $\mathrm{H}_{2} \mathrm{O}$ and PBS and blocked with 20\% fetal calf serum in PBS for 20 minutes. The specimens were exposed to the primary antibody ( $1 / 250$ dilution in PBS), for one hour at room temperature. Subsequently, the tissue samples were washed with $\mathrm{H}_{2} \mathrm{O}$, followed by biotinylated swine antirabbit immunoglobulin (1/300 dilution; Vector Laboratories, Burlingame, California, USA), and finally avidin-biotin complex (Dako). The peroxidase reaction was then performed using 3,3'diaminobenzidine (Sigma Chemical Co). The slides were counterstained with haematoxylin for 15 seconds, rehydrated with increasing concentrations of ethanol and xylene, and mounted.

For control purposes, several staining methods were compared. The avidin-biotin method and the streptavidin method on paraffin wax embedded sections all gave similar results. Primary antibody staining times, varying from 30 minutes to 24 hours, at both room temperature and $+4^{\circ} \mathrm{C}$, were tested. Permeabilisation was carried out using different enzyme pretreatment protocols, various concentrations,

Table 1 Number of lesions and vessels measured by computer assisted image analysis

\begin{tabular}{llll}
\hline Type of lesion & $\begin{array}{l}\text { Number of } \\
\text { lesions }\end{array}$ & $\begin{array}{l}\text { Number of } \\
\text { vessels }\end{array}$ & $\begin{array}{l}\text { Mean vessel density } \\
\left(/ \mathrm{mm}^{2}\right)\end{array}$ \\
\hline Normal & 15 & 202 & 70 \\
Mild dysplasia & 10 & 155 & 81 \\
Severe dysplasia & 5 & 223 & 232 \\
SCC grade I & 10 & 248 & 129 \\
SCC grade II & 10 & 222 & 116 \\
SCC grade III & 10 & 421 & 219 \\
Total & 60 & 1451 & 126 \\
\hline \\
The mean vessel density is the total number of vessels divided by the total \\
area measured in mm ${ }^{2}$. \\
SCC, squamous cell carcinoma.
\end{tabular}

durations, and temperatures, in addition to microwave pretreatment using different temperatures and durations; pretreatment by boiling was also investigated. To exclude non-specific staining, the following controls were set up: (a) second antibody only; (b) replacing the first antibody with saline or non-reactive serum; and (c) replacing the reactive compound in the various steps with a non-reactive one, or omitting the step completely. Specimens of human bronchi, lung, skin, and skin tumours, in addition to specimens from cultures of human and animal epidermal cells, were also used as controls.

\section{Automated image analysis}

To assess vessel involvement, the number, size, shape, structure, and location of vessels in the stroma were determined using quantitative densitometry and morphometry. Details of the method have been published previously. ${ }^{30}$ We used a CAS200 (Becton-Dickinson, Leiden, The Netherlands) automated image analyser and the proprietary software. The specimens were analysed and classified on the basis of absorbance analysis, using two simultaneously recorded video camera images processed by computer, and using standard software. Immunoreactivity was measured based on the assumption that the total absorbance was directly proportional to immunoreactivity. Applying the Lambert-Beer law of spectral overlap and linearity, using applicable calibration and controls, yielded appropriate linearity between light and dark settings. The intensity was measured at 500 and $620 \mathrm{~nm}$. Each measurement consisted of 65536 pixels, approximating $320 \times 600 \mu \mathrm{m}$, which comprised approximately $50 \%$ of the stroma adjacent to the epithelial component in the specimen.

The number of vessels was determined by computer based image analysis of vessels with positive FVIII staining exceeding a preset threshold value. The preset threshold was based on the intensity of immunostaining of the vessels, with maximum sensitivity of vessel staining and minimum non-specific background being the only criteria, as shown in a previous study. ${ }^{30}$ The procedure was carried out using a modification of the proprietary cell measurement computer program (CMP) as follows: a filter was designed for automatic vessel classification allowing analysis of individual vessels. Size classes were: small vessels, $16-300 \mu \mathrm{m}^{2}$ in cross sectional area; intermediate vessels, between $300 \mu \mathrm{m}^{2}$ and $2800 \mu \mathrm{m}^{2}$ in area; and large vessels, above $2800 \mu \mathrm{m}^{2}$ in area. The appropriate background light intensity was calibrated, as were corresponding antibody values. Measurements were recorded for vessels fulfilling the morphological criteria; thus, contaminating populations were excluded. Vessel size was measured directly by computer. Vessel shape (the shape factor determining vessel irregularity) was based on the ratio of perimeter ${ }^{2}$ to cell size. The staining intensity of FVIII was based on measurements of average optical density. Repeated measurements showed accuracy exceeding $0.01 \mu \mathrm{m}$ and sensitivity and reproducibility exceeding 99\%. The results were based on repeated measurements of the same field, in addition to variability between groups; the method has been described previously. ${ }^{30}$

The results are presented as number of vessels/field in normal tissue, dysplasia, and carcinoma, in different histopathological groups, and in different groups divided according to size. Characteristics of individual vessels in different histopathological groups were also measured. Ninety five per cent confidence intervals for means of vessel size, shape, and staining intensity were calculated, and examples of the distribution of absolute values are presented: vessel size, shape, and staining intensity. Evaluation of vessel direction in relation to the direction of the BM was carried out by calculating the ratio of vessel length perpendicular to the BM 
to that in the direction of the BM. Characteristics of individual vessels were also compared; these included size in relation to shape and staining intensity, and shape in relation to staining intensity. For each vessel the relation of size to shape, to sum of optical density and to average optical density; the relation of shape to size, to sum of optical density and to average optical density; the relation of sum of optical density to size, to shape, and to average optical density; and the relation of average optical density to size, to shape, and to sum of optical density is obtained from the location of each dot on the $\mathrm{x}$ and $\mathrm{y}$ axes.

\section{Statistical methods}

Statistical analysis of vessel characteristics was performed using the SPSS for Windows Release 8.0.1 (SPSS $^{\odot}$ Inc, Chicago, Illinois, USA). The significance of number of vessels was estimated using one sample $t$ tests to compare all lesions versus normal tissue. To assess individual vessel characteristics (size, shape, intensity of FVIII staining, and area of positive immunoreactivity), the mean, SD, and 95\% confidence intervals for means of computer derived values were calculated. The significance of the measurements was estimated by the following sequence: one sample Kolmogorov-Smirnov tests were used to test the normality of the samples, and mean, variance, kurtosis, and skewness were examined. Thus, square root transformation was performed, giving new variables used for one way ANOVA. Large sample sizes justified one way ANOVA by the central limit theorem. Levene's test was used to test for homogeneity of variances, and because the variances were found to be unequal, Dunnett's T3 test was used for post hoc pairwise analysis. Spearman's coefficient $\rho$ was used to assess the correlation between the extent of FVIII staining and the stages and types of dysplasia and tumour.

Vessel size, vessel shape, and staining intensity were similarly studied. Levene's test was used to test for homogeneity of variances, and because the variances were found to be unequal, Dunnett's T3 test was used for post hoc pairwise analysis.

The ratio of vessel length perpendicular to the BM to vessel length in the direction of the BM was used to calculate vessel directional angle. Tests for normality were carried out using the one sample Kolmogorov-Smirnov test. With normality tests passed, one way ANOVA and the Kruskall-Wallis H test were used. When Levene's test for homogeneity of variance was significant, Dunnett's T3 test was used for post hoc pairwise analysis.

\section{RESULTS}

The vascular structure in stroma adjacent to unaltered squamous epithelium consisted of numerous FVIII positive vessels in the extracellular matrix (ECM). The subepithelial vessels were regular, branched (fig 1), and mostly located close to the epithelium. The numbers of FVIII stained vessels are shown in fig 2A. Most vessels belonged to the small sized group, below $300 \mu \mathrm{m}^{2}$ (fig $2 \mathrm{~B}$ ). Vessel size (fig 3A) averaged $248 \mu^{2}$; vessel shape (fig $3 \mathrm{~B}$ ), ratio of perimeter ${ }^{2}$ to vessel size, was 19.4; and staining intensity (fig 3C), measured as average optical density, was 0.463 .

Increased angiogenesis in preneoplastic conditions (dysplasia) was seen as an increase in vessel number (fig 2A). In computer assisted image analysis, the vessels were small, round, and located close to the epithelial layer. In mild dysplasia, we found no significant differences in vessel number, size, (fig 3A), shape (fig 3B), or staining intensity (fig 3C), compared with normal tissue. Moderate dysplasia was not analysed for vascular alterations because of difficulties in reproducible classification caused by adjacent epithelial mild or severe dysplasia. In severe dysplasia, the vascular structures were prominent, enlarged, and branched (fig 1B). Morphometric analysis of vessel number (fig 2) showed a significant increase compared with normal tissue $(\mathrm{p}<0.01)$; this was also related to intermediate and large sized vessels (fig 2B). In severe dysplasia, there was a difference in the distribution of different sized vessels (fig 4B) compared with normal tissue (fig 4A).

Increased angiogenesis in squamous cell carcinomas was seen as increased numbers of vessels (fig 2A). Depending upon the degree of differentiation, three different patterns of vessel organisation were seen (fig $\mathrm{lC}-\mathrm{F}$ ). In well differentiated (grade I) squamous cell carcinomas the vessels were arranged in a circumferential pattern (fig $1 C$ ), in the same direction as the BM. This was supported by computer assisted image analysis of vessel direction, with numerous vessels in the same direction as the $\mathrm{BM}$, an angle of near $0^{\circ}$, and few vessels perpendicular to the BM (fig 5). The ECM contained an increased number of both intermediate size and large vessels. The average vessel size (fig $3 \mathrm{~A}$ ) was similar to that seen in normal epithelium or in mild dysplasia, being significantly smaller than that seen in severe dysplasia (table $2 ; \mathrm{p}<0.006$ ). There was a significant increase in vessel shape alterations (fig 3B) compared with normal tissue (table $2 ; \mathrm{p}<0.046$ ). FVIII staining intensity was significantly decreased (fig 3C) compared with normal tissue (table 2; p $<0.003$ ).

Increased angiogenesis in moderately differentiated (grade II) squamous cell carcinomas also involved mainly intermediate and large vessels. In these tumours, vessel organisation was that of directional angiogenesis, with vessels growing in the ECM in the direction of the BM, and a few tangential to the BM (fig 1D-F). Computer assisted image analysis of vessel direction (fig 5) showed many vessels growing in the direction of the BM. Altered vessel structure was reflected in increased vessel size (fig 3A) compared with normal. The mean vessel shape was increased (fig 3B) compared with preneoplasia and more differentiated squamous cell carcinomas, and the distribution of the vessel shape ratio was also different (fig 4C, D).

Aberrant angiogenesis-an increased number of irregular vascular structures-was prominent in poorly differentiated (grade III) squamous cell carcinomas. In these neoplasms, tumour cells with pronounced cytological abnormalities were growing in cords and small fascicles deep in the surrounding stroma. Vascular proliferation was distinct, consisting of numerous tortuous vessels crisscrossing the stroma. Vessel direction was mixed-no directional or circumferential angiogenesis was seen. The number of vessels (fig $2 \mathrm{~B}$ ) was increased significantly compared with normal $(\mathrm{p}<0.001)$, mild dysplasia $(p=0.001)$, and grade I squamous cell carcinoma $(p=0.022)$. Altered vessel structures were reflected in increased vessel size and altered shape compared with normal. The mean FVIII staining intensity was significantly decreased in grade III squamous cell carcinomas (fig 3C) compared with all other morphological entities $(\mathrm{p}<0.001)$. The distribution of FVIII staining was also different (fig 4E, F).

Quantitative estimates of the numbers of vessels in different histological states (fig 2) were also analysed statistically. Tests for normality were carried out using the one sample Kolmogorov-Smirnov test, examining the mean, variance, skewness, and kurtosis. Mean and variance were positively correlated. This was confirmed by plotting mean against variance, resulting in $r^{2}=0.6395(\mathrm{p}<0.05)$. Thus, square root transformation was performed, giving new variables used for one way ANOVA. This analysis showed a significant difference between group means $(p<0.0005)$. With Levene's test for homogeneity of variance being significant $(\mathrm{p}=0.009)$, Dunnett's T3 test was used for post 

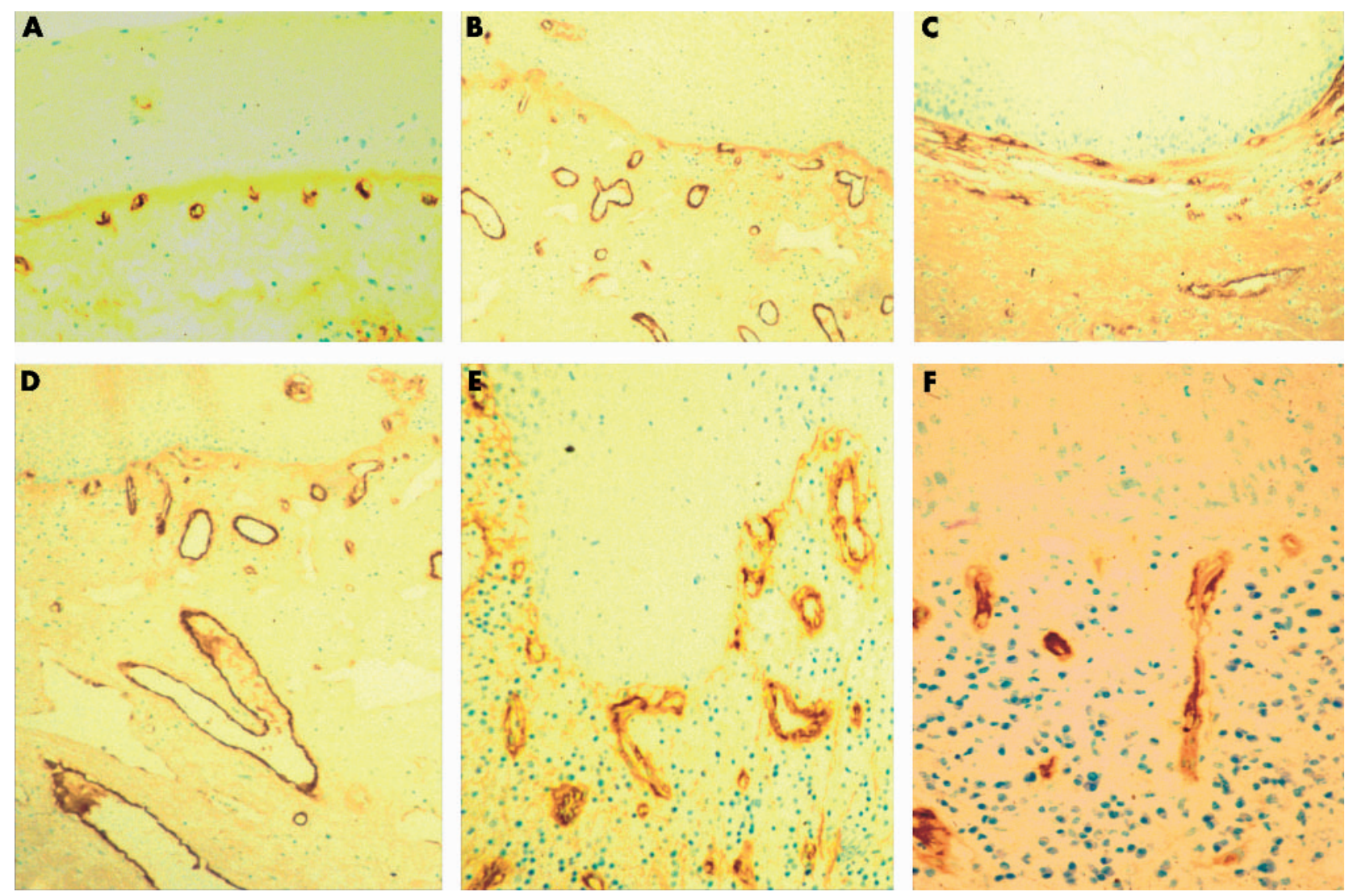

Figure 1 Laryngeal preneoplastic and neoplastic conditions stained by antibodies to factor VIII. (A) Vessels in the stroma of unaltered epithelium. (B) Severe epithelial dysplasia with prominent blood vessels. (C) Well differentiated squamous cell carcinoma with vessels in circumferential arrangements. (D-F) Moderately differentiated laryngeal squamous cell carcinoma with vessels extending from the stroma in the direction of the epithelial basement membrane (directional angiogenesis).

hoc pairwise analysis. Probability values thereby obtained were significant for severe dysplasia versus normal $(\mathrm{p}=0.014)$, in addition to undifferentiated squamous cell carcinoma versus normal $(\mathrm{p}<0.0005)$ and versus mild dysplasia $(\mathrm{p}<0.001)$.

The direction of vessel growth in the different morphological lesions was investigated by calculating the ratio of vessel length perpendicular to the BM to that in the direction of the BM. Figure 5 shows the direction of vessels in relation to the direction of the BM expressed in degrees. Low values indicate that the predominant direction is along the BM; high values indicate that the predominant vessel direction is perpendicular to the BM. In severe dysplasia, the average vessel direction was $54^{\circ}$, in well differentiated squamous cell carcinomas it was $48^{\circ}$, and in moderately well differentiated squamous cell carcinomas it was $39^{\circ}$. One way ANOVA showed significant differences between the groups $(p<0.0005)$. Dunnett's T3 test was used for post hoc pair wise analysis. Table 3 shows the significant pairs.

Comparison of vessel characteristics (size, shape, and staining intensity) in individual vessels showed correlations between the various parameters. Increased size was associated with increasing shape alterations, although varying in extent and related to degree of malignancy. Increased size was directly associated with total staining intensity regardless of histopathological diagnosis. Similarly, increased size was associated with increased average staining intensity. Altered shape was also associated with increased total and average staining intensity, although varying in extent and related to the histopathological diagnosis. The findings were analysed statistically. One way ANOVA showed significant differences between group means for size $(p=0.002)$, shape $(p=0.018)$, and optical density $(p=0.002)$. The KruskallWallis $\mathrm{H}$ test gave the following significance values: size, $p=0.016$; shape, $p=0.043$; and optical density, $\mathrm{p}<0.0005$. FVIII staining intensity increased with increasing vessel size, with Pearson's two tailed correlation being significant at $\mathrm{p}<0.01$. FVIII staining intensity also increased with increased alterations in vessel shape, with Pearson's two tailed correlation being significant at $\mathrm{p}<0.01$. Shape alterations also increased with increased vessel size, with Pearson's two tailed correlation being significant at $\mathrm{p}<0.01$.

\section{DISCUSSION}

Increased angiogenesis was a hallmark of laryngeal preneoplasia and neoplasia in this study. Angiogenesis was reflected as an increase in the total number of vessels and correlated with malignant transformation (fig 2). Angiogenesis is commonly investigated using the "hot spot" counting method. ${ }^{9-16}$ However, angiogenic hot spots do not always define high vascularisation. ${ }^{33}$ Assessments of vessel counts in histological sections are two dimensional, influenced by coiling, tortuosity, and compression of vessels. ${ }^{34}$ The selection of maximum vascularisation in the "hot spot" approach is prone to error because of observer bias. ${ }^{34}$ Angiogenesis was also reflected by increased vessel volume in malignant states (fig 3A). Vessel volume/volume of tumour tissue, as derived from the measurement of vascular surface density, may constitute a better estimate of angiogenesis. ${ }^{35}$ Formation of new vessels may be more related to 

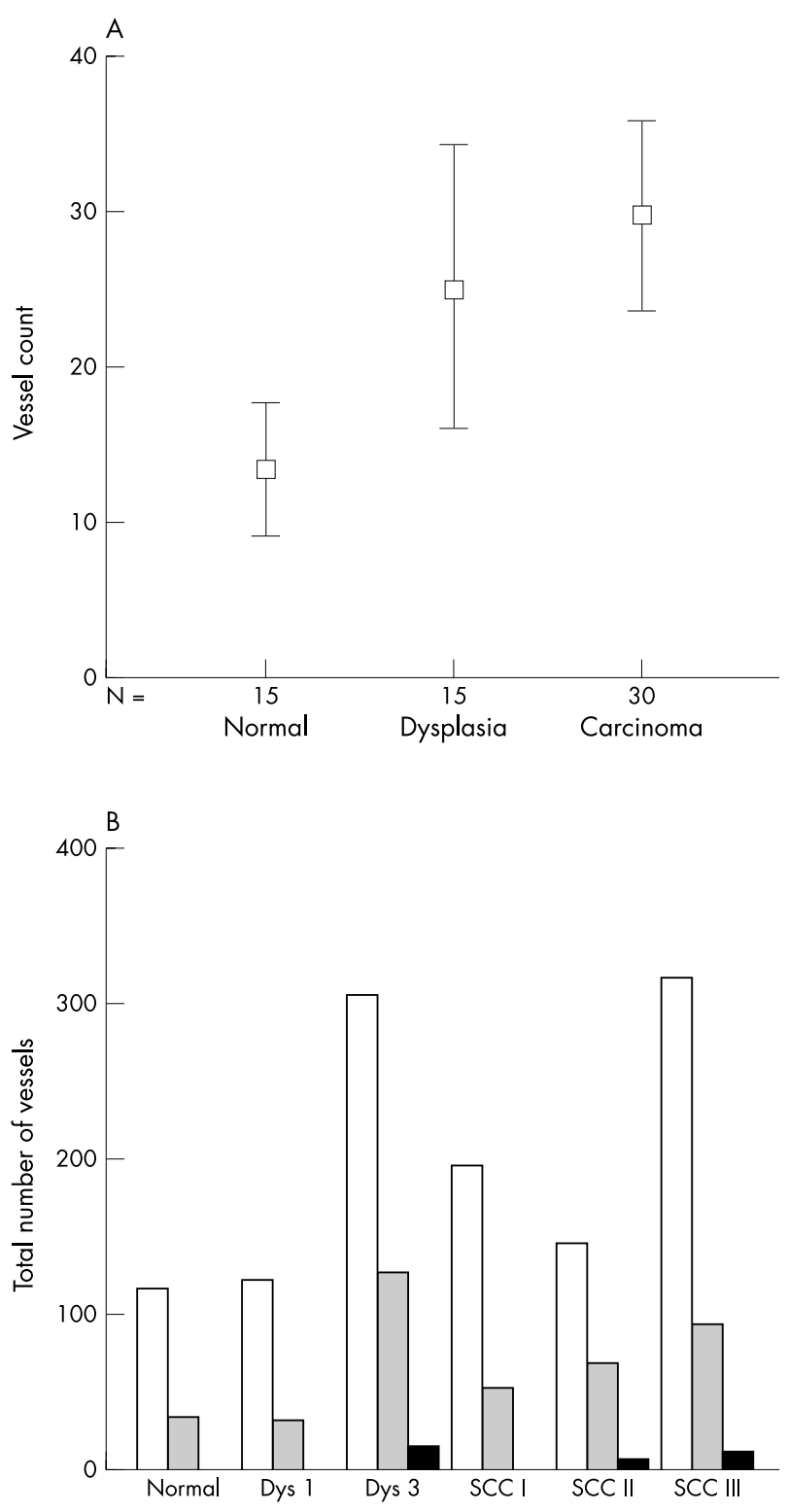

Figure 2 (A) Automated quantitative image analysis of vessel count in each measurement area of factor 8 stained vessels in specimens of laryngeal normal tissue, dysplasia, and squamous cell carcinoma; means and $95 \%$ confidence intervals are shown. $N$, number of vessels analysed. (B) Total numbers of vessels in normal tissue, mild (Dys 1) and severe dysplasia (Dys 3), and squamous cell carcinoma (SCC) grades I, II, and III divided according to size. Open bars, 16-300 $\mu \mathrm{m}$; shaded bars, 301-2800 $\mu \mathrm{m}$; black bars, $>2801 \mu \mathrm{m}$.

vessel number, whereas vessel volume may be used to assess functionally active vessels.

Vessel structure characteristics were also dependent upon tumour morphology in our study (fig 2). Small, regular vessels were common in benign lesions and large, irregular vessels in malignant neoplasms (fig 3). Point counting to determine microvessel volume was more informative than the analysis of microvessel density in a study of tumour associated angiogenesis. ${ }^{35}$ In normal tissue and slowly growing, low grade tumours, the microvessels were well branched and showed a high surface to vessel ratio, whereas the microvessels in high grade, highly malignant lesions were plump and non-branched, showing a low surface to vessel ratio. ${ }^{34}$ Estimates of vascular surface density
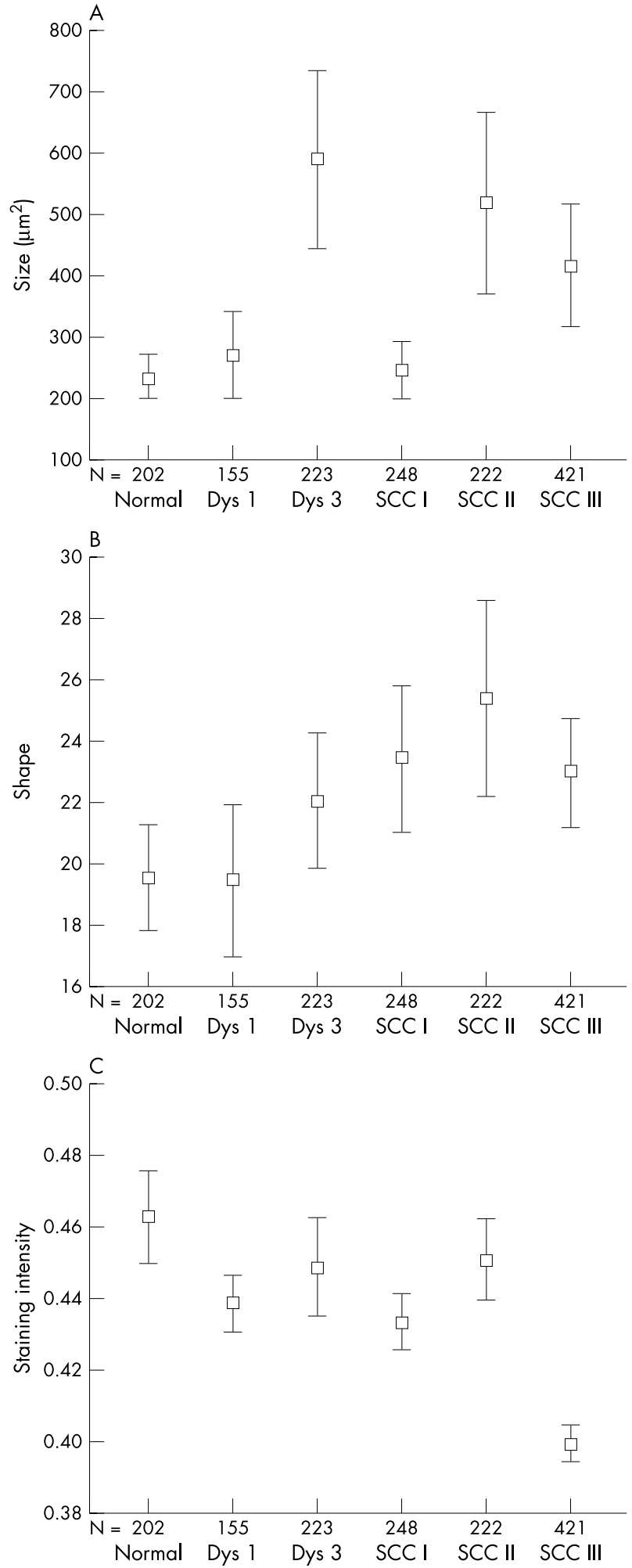

Figure 3 Measurements of factor VIII (FVIII) stained vessels in normal tissue, mild (Dys 1) and severe dysplasia (Dys 3), and squamous cell carcinoma (SCC) grades I, II, and III, as determined by automated quantitative image analysis and expressed as error bars with mean and $95 \%$ confidence intervals. N, number of vessels analysed. (A) Individual vessel size in $\mu \mathrm{m}^{2}$. (B) Individual vessel shape, perimeter ${ }^{2} /$ vessel area. (C) FVIII staining intensity (average optical density).

should involve precise measurement of vessel area/volume of tumour tissue, difficult in practice because of sample variation. 

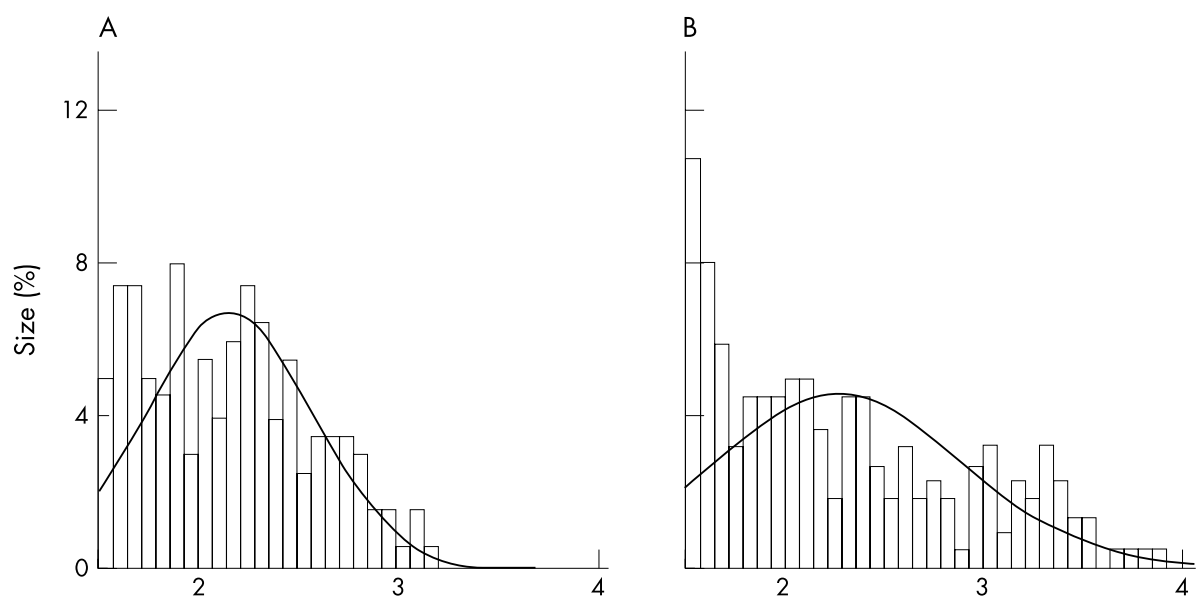

Figure 4 Distribution of logarithmic measurements from automated quantitative image analysis of factor VIII (FVIII) stained specimens. (A) Distribution of vessel size in normal epithelium; (B) distribution of vessel size in severe dysplasia. (C) Shape distribution in normal epithelium; (D) shape distribution in moderately differentiated squamous cell carcinoma. (E) Distribution of FVIII staining intensity in normal epithelium; (F) distribution of FVIII staining intensity in less well differentiated squamous cell carcinoma.
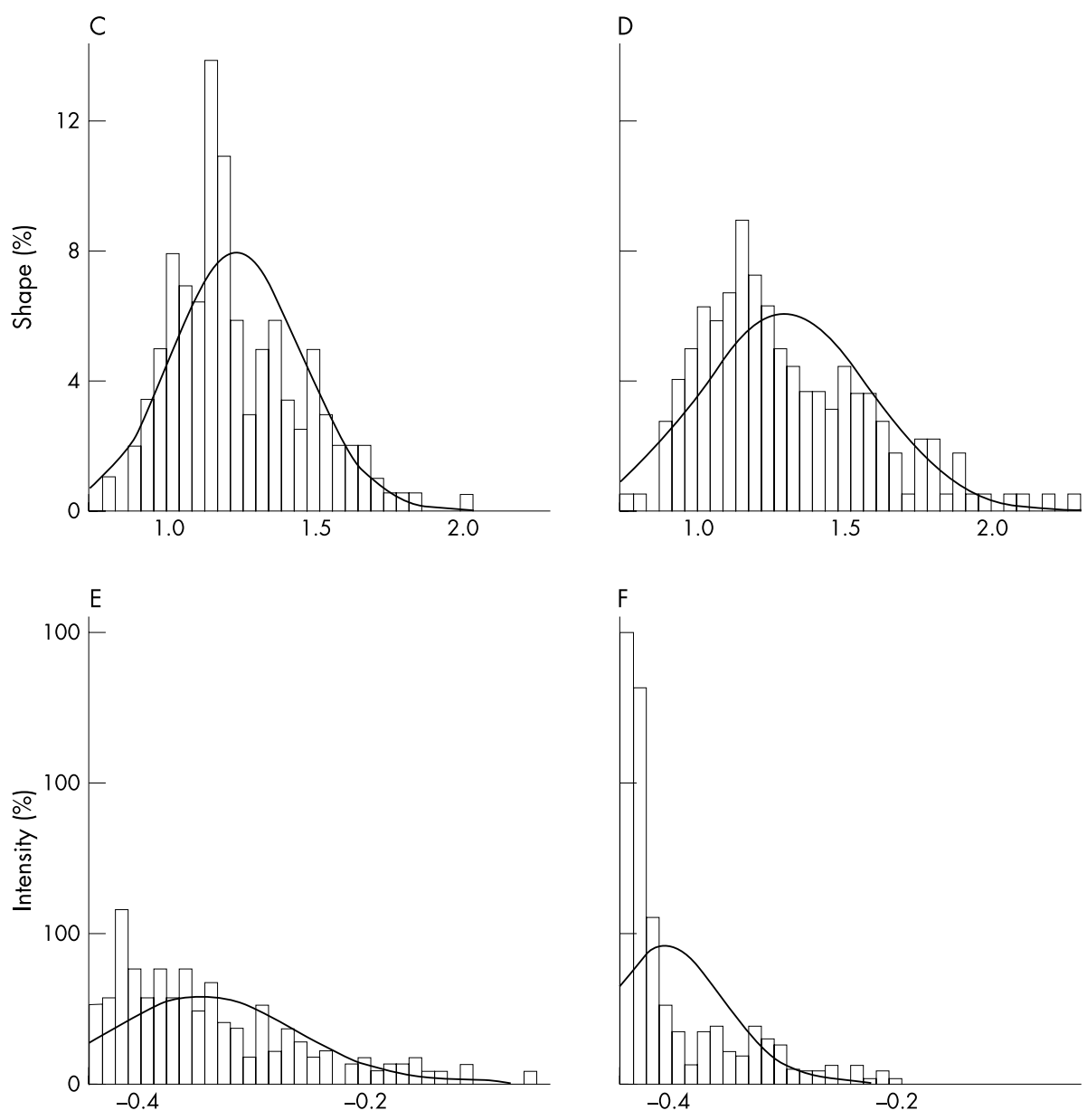

Increased vessel number and vessel size occurred before malignant transformation and invasion in our study. Studies on stage related angiogenesis in experimental animals suggest that tumour progression requires a switch from a prevascular to an angiogenic phase in the transition from hyperplasia to neoplasia. ${ }^{36}$ Intraepithelial melanomas, dormant tumours, and carcinomas in situ are usually avascular, and to grow progressively they must induce nearby capillaries to develop a new vascular network around and within the tumour. ${ }^{37}$ In cervical dysplasia, the induction of microvessels is significantly increased in high grade versus low grade lesions, ${ }^{38}$ and a similar phenomenon was also seen here.

\section{"Increased vessel number and vessel size occurred before malignant transformation and invasion in our study"}

Vascular organisation was distinctly dependent on the degree of laryngeal tumour differentiation in our study. In well differentiated squamous cell carcinomas, the vessels extend in the direction of the BM, here termed "circumferential angiogenesis". The explanation is possible compression of the ECM by expanding tumour tissue. In moderately differentiated squamous cell carcinomas, the vessels extend in a direction perpendicular to the BM, here termed "directional angiogenesis". The production of angiogenic substances $^{39}$ is probably important. Angiogenesis in squamous 


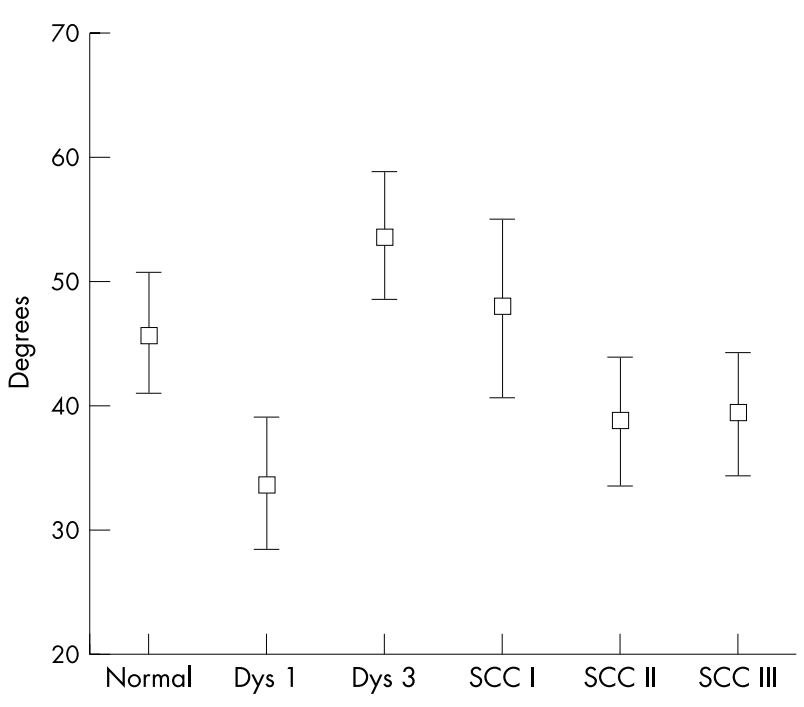

Figure 5 Automated quantitative image analysis of factor VIII stained specimens of laryngeal lesions describing the direction of the vessel in relation to the direction of the basement membrane expressed in degrees. Results are shown as error bars, with mean and $95 \%$ confidence intervals. Dys 1, mild dysplasia; Dys 3, severe dysplasia; SCC, squamous cell carcinoma.

Table 2 Significant differences in individual vessel size, shape, and staining intensity in laryngeal squamous cell carcinoma and preneoplastic lesions

\begin{tabular}{llll}
\hline Marker & Morphology & & p Value \\
\hline Size & Severe dysplasia & SCC grade I & 0.006 \\
Shape & Normal & SCC grade I & 0.046 \\
Staining intensity & Normal & SCC grade I & 0.003 \\
& Normal & SCC grade III & $<0.0005$ \\
& Mild dysplasia & SCC grade III & $<0.0005$ \\
& Severe dysplasia & SCC grade III & $<0.0005$ \\
& SCCC grade 1 & SCC grade III & $<0.0005$ \\
& SCC grade 2 & SCC grade III & $<0.0005$ \\
\hline
\end{tabular}

SCC, squamous cell carcinoma.

cell carcinoma of the oesophagus was associated with the tumour growth pattern-that is, flat, expansive, or a downgrowth pattern. ${ }^{40}$ In studies measuring the distance between tumour cells and vessels, a short distance was associated with a worse prognosis. ${ }^{13}$ In rapidly growing, less differentiated, highly malignant neoplasms, an increased number

\section{Take home messages}

- Increased angiogenesis occurred early in the development of laryngeal tumours-vessel numbers were increased in preneoplastic states and severe dysplasia, in addition to squamous cell carcinomas

- Vessel structure, size, distribution, and shape were related to tumour growth pattern and behaviour

- Small regular vessels predominated in benign conditions and large, irregular vessels in malignant neoplasms

- In the future, automated image analysis may help predict clinical outcome and aid in defining preneoplastic and neoplastic lesions requiring aggressive treatment
Table 3 Significant differences in angle relative to basal membrane in laryngeal squamous cell carcinoma and preneoplastic lesions

\begin{tabular}{llll}
\hline Marker & Morphology & & p Value \\
\hline Vascular angle & Normal & Mild dysplasia & 0.017 \\
& Mild dysplasia & Severe dysplasia & $<0.0005$ \\
& Mild dysplasia & SCC grade I & 0.032 \\
Severe dysplasia & SCC grade II & 0.001 \\
& Severe dysplasia & SCC grade III & 0.002 \\
\hline \multirow{2}{*}{ SCC, squamous cell carcinoma. } & & \\
\end{tabular}

of irregular vascular structures was seen, here termed "aberrant angiogenesis". This pattern may be related to vasculogenic mimicry, a recently described entity in uveal melanoma angiogenesis. ${ }^{41}$ Structural alterations occur in vessels in laryngeal cancers, with newly formed capillaries having embryonic characteristics, varying from single, blind ended pipes, via long hairpin loops, to strongly convoluted loops resembling pseudoglomeruli. ${ }^{42}$

When comparing the characteristics of the individual vessels - their size, shape, and staining intensity-we found that decreased FVIII staining intensity was associated with increased degree of malignancy. Other studies have shown different results, perhaps as a result of variations in detection method sensitivity. ${ }^{8} 11-131819124344$ Immunohistochemical staining results are affected by antibody selection, staining methods, and tissue preparation. ${ }^{30}$ FVIII is a specific marker of endothelial cells, ${ }^{45}$ and other investigators ${ }^{35}$ have reported similar results on angiogenesis when using either anti-FVIII or anti-CD31 antibodies. ${ }^{9} 10212445$ The CD34 antibody has also been used as a vessel marker. ${ }^{15} 1620$ FVIII staining intensity increased with increased vessel size in our study. FVIII related antigen may be a better marker for mature vessels than immature vessels; this might account for the more intense staining of larger vessels, but may also mean that new, small vessels are not assessed by this method.

Angiogenesis is an independent predictor of disease free survival in patients with laryngeal carcinoma, ${ }^{8-10} 12-1543$ although conflicting results have been reported. ${ }^{16-2444} 45$ The clinical outcome of the treatment of oral squamous cell carcinomas correlates with different patterns of tumour architecture and blood supply. ${ }^{13}$ Tumour oxygenation and vasculature were determinants for radiotherapy outcome and prognosis in patients with squamous cell carcinomas of the head and neck. ${ }^{46}$ Angiogenesis - the formation of new vessels-is related to vessel number, whereas vessel volume can be used to assess functionally active vessels. Increased vascularity in relation to severe dysplasia has been described previously in oral lesions..$^{13}$ although whether this is dilatation of existing vessels or new vessel formation, true angiogenesis, is unclear. Different types of vessels may have different relevance to treatment; whether the larger or smaller vessels are more important in the delivery of oxygen to the tissues and which vessels are more likely to be targets for antiangiogenic treatment are presently unclear. In the future, automated image analysis ${ }^{29} 3047$ may help predict clinical outcome and aid in defining preneoplastic and neoplastic lesions requiring aggressive treatment.

\section{ACKNOWLEDGEMENTS}

The authors gratefully acknowledge the expert technical assistance in immunohistochemistry of Mrs R Vuento, Mrs T Lujala, Mrs E Tomperi, and Mrs M Vahera; the photography and computer analysis carried out by Mr H Väänänen and Mr T Leinonen; and the statistical analysis carried out by Dr D Harrison. The financial support of Oulu University Hospital, the Finnish Cancer Society, and Finska Läkarsällskapet, Finland, is gratefully acknowledged. 
Authors' affiliations

J Laitakari, V Näyhä, F Stenbäck, Department of Pathology, University of Oulu, PO Box 5000, Oulu 90014, Finland

\section{REFERENCES}

1 Manni JJ, Terhaard $\mathrm{CH}$, de Boer MF, et al. Prognostic factors for survival in patients with T3 laryngeal carcinoma. Am J Surg 1992;164:682-7.

2 Bradford CR, Wolf GT, Fisher SG, et al. Prognostic importance of surgical margins in advanced laryngeal squamous carcinoma. Head Neck 1996;18:11-16.

3 Pera E, Moreno A, Galindo L. Prognostic factors in laryngeal carcinoma. A multifactorial study of 416 cases. Cancer 1986;58:928-34.

4 Stell PM. Prognosis in laryngeal carcinoma: tumour factors. Clin Otolaryngol 1990;15:69-81.

5 Eiband JD, Elias EG, Suter CM, et al. Prognostic factors in squamous cell carcinoma of the larynx. Am J Surg 1989;158:314-17.

6 McLaren KM, Burnett RA, Goodlad JR, et al. Consistency of histopathological reporting of laryngeal dysplasia. The Scottish Pathology Consistency Group. Histopathology 2000;37:460-3.

7 Guerry M, Vabre L, Talbot M, et al. Prognostic value of histological and biological markers in pharyngeal squamous cell carcinoma: a case-control study. Br J Cancer 1998;77:1932-6.

8 Williams JK, Carlson GW, Cohen C, et al. Tumor angiogenesis as a prognostic factor in oral cavity tumors. Am J Surg 1994;168:373-80.

9 Murray JD, Carlson GW, McLaughlin K, et al. Tumor angiogenesis as a prognostic factor in laryngeal cancer. Am J Surg 1997; 174:523-6.

10 Beatrice F, Cammarota R, Giordano C, et al. Angiogenesis: prognostic significance in laryngeal cancer. Anticancer Res 1998;18:4737-40.

11 Albo D, Granick MS, Jhala N, et al. The relationship of angiogenesis to biological activity in human squamous cell carcinomas of the head and neck. Ann Plast Surg 1994;32:588-94.

12 Kupisz K, Chibowski D, Klatka J, et al. Tumor angiogenesis in patients with laryngeal cancer. Eur Arch Otorhinolaryngol 1999;256:303-5.

13 Lauk S, Skates S, Goodman M, et al. A morphometric study of the vascularity of oral squamous cell carcinomas and its relation to outcome of radiation therapy. Eur J Cancer Clin Oncol 1989;25:1431-40.

14 Sauter ER, Nesbit M, Watson JC, et al. Vascular endothelial growth factor is a marker of tumor invasion and metastasis in squamous cell carcinomas of the head and neck. Clin Cancer Res 1999;5:775-82.

15 Sawatsubashi M, Yamada T, Fukushima N, et al. Association of vascular endothelial growth factor and mast cells with angiogenesis in laryngeal squamous cell carcinoma. Virchows Arch 2000;436:243-8.

16 Tae K, El-Naggar AK, Yoo E, et al. Expression of vascular endothelial growth factor and microvessel density in head and neck tumorigenesis. Clin Cancer Res 2000;6:2821-8.

17 Leedy DA, Trune DR, Kronz JD, et al. Tumor angiogenesis, the p53 antigen, and cervical metastasis in squamous carcinoma of the tongue. Otolaryngol Head Neck Surg 1994;111:417-22.

18 Zatterstrom UK, Brun E, Willen R, et al. Tumor angiogenesis and prognosis in squamous cell carcinoma of the head and neck. Head Neck 1995;17:312-18.

19 Dray TG, Hardin NJ, Sofferman RA. Angiogenesis as a prognostic marker in early head and neck cancer. Ann Otol Rhinol Laryngol 1995;104:724-9.

20 Burian M, Quint C, Neuchrist C. Angiogenic factors in laryngeal carcinomas: do they have prognostic relevance? Acta Otolaryngol 1999; 1 19:289-92.

21 Gleich LL, Biddinger PW, Pavelic ZP, et al. Tumor angiogenesis in T1 oral cavity squamous cell carcinoma: role in predicting tumor aggressiveness. Head Neck 1996;18:343-6

22 Neuchrist C, Quint C, Pammer A, et al. Vascular endothelial growth factor (VEGF) and microvessel density in squamous cell carcinomas of the larynx: an immunohistochemical study. Acta Otolaryngol 1999;119:732-8.

23 Penfold CN, Partridge $M$, Rojas $R$, et al. The role of angiogenesis in the spread of oral squamous cell carcinoma. Br J Oral Maxillofac Surg 1996;34:37-41.
24 Moriyama M, Kumagai S, Kawashiri S, et al. Immunohistochemical study of tumour angiogenesis in oral squamous cell carcinoma. Oral Oncol 1997;33:369-74.

25 Jenssen N, Boysen M, Kjaerheim A, et al. Low vascular density indicates poor response to radiotherapy in small glottic carcinomas. Pathol Res Pract 1996; 192:1090-4

26 Salven P, Heikkilä P, Anttonen A, et al. Vascular endothelial growth factor in squamous cell head and neck carcinoma: expression and prognostic significance. Mod Pathol 1997; 10:1 128-33.

27 Kramer RH, Vogel KG, Nicolson GL. Tumor cell interactions with vascular endothelial cells and their extracellular matrix. Progr Clin Biol Res 1982;89:333-51

28 Gasparini G, Weidner N, Maluta S, et al. Intratumoral microvessel density and p53 protein: correlation with metastasis in head-and-neck squamous-cell carcinoma. Int J Cancer 1993;55:739-44.

29 Lohmann CM, Fields AC, Schiller A, et al. Quantitation of angiogenesis by measuring CD 31 and factor VIIII visually and by image cytometry in renal cell carcinomas - prognostic significance. J Urol Pathol 1999;11:101-112.

30 Laitakari J, Harrison D, Stenbäck F. Morphometrical characteristics of cell proliferation and p53 expression in development of experimentally induced respiratory tumors. Analyt Quant Cytol Histol 2001;23:273-86.

31 Vermeulen PB, Gasparini G, Fox SB, et al. Quantification of angiogenesis in solid human tumours: an international consensus on the methodology and criteria of evaluation. Eur J Cancer 1996;32A:2474-84.

32 Hyams VJ, Batsakis JG, Michaels L, eds. Atlas of tumor pathology. Washington, DC: Armed Forces Institute of Pathology, 1988.

33 Giatromanolaki A, Koukourakis MI, Kakolyris S, et al. Vascular endothelial growth factor, wild-type p53, and angiogenesis in early operable non-small cell lung cancer. Clin Cancer Res 1998;4:3017-24.

34 Barth PJ, Weingartner K, Kohler HH, et al. Assessment of the vascularisation in prostatic carcinoma: a morphometric investigation. Hum Pathol 1996;27:1306-10.

35 Pazouki S, Chisholm DM, Adi MM, et al. The association between tumour progression and vascularity in the oral mucosa. J Pathol 1997;183:39-43.

36 Bergers G, Hanahan D, Coussens LM. Angiogenesis and apoptosis are cellular parameters of neoplastic progression in transgenic mouse models of tumorigenesis. Int J Dev Biol 1998;42:995-1002.

37 Barnhill RL, Fandrey K, Levy MA, et al. Angiogenesis and tumor progression of melanoma. Quantification of vascularity in melanocytic nevi and cutaneous malignant melanoma. Lab Invest 1992;67:331-7.

38 Guidi AJ, Abu-Jawdeh G, Berse B, et al. Vascular permeability factor (vascular endothelial growth factor) expression and angiogenesis in cervical neoplasia. J Natl Cancer Inst 1995;87:1237-45.

39 Dano K, Andreasen PA, Grondahl-Hansen J, et al. Plasminogen activators, tissue degradation, and cancer. Adv Cancer Res 1985;44:139-266.

40 Kuwano H, Sonoda K, Yasuda M, et al. Tumor invasion and angiogenesis in early esophageal squamous cell carcinoma. J Surg Oncol 1997;65:188-93.

41 Folberg R, Hendrix MJ, Maniotis AJ. Vasculogenic mimicry and tumor angiogenesis. Am J Pathol 2000;156:361-81.

42 Kus J, Miodonski A, Olszewski E, et al. Morphology of arteries, veins, and capillaries in cancer of the larynx: scanning electron-microscopical study on microcorrosion casts. J Cancer Res Clin Oncol 1981;100:271-83.

43 Sion-Vardy N, Fliss DM, Prinsloo I, et al. Neoangiogenesis in squamous cell carcinoma of the larynx-biological and prognostic associations. Pathol Res Pract 2001;197:1-5.

44 Lukits J, Timar J, Juhasz A, et al. Progression difference between cancers of the larynx and hypopharynx is not due to tumor size and vascularization. Otolaryngol Head Neck Surg 2001;125:18-22.

45 Hagedorn HG, Nerlich AG. Microvessel density and endothelial basement membrane composition in laryngeal squamous cell carcinomas. Acta Otolaryngol 2000;120:891-8.

46 Wijffels KI, Kaanders JH, Rijken PF, et al. Vascular architecture and hypoxic profiles in human head and neck squamous cell carcinomas. Br J Cancer 2000:83:674-83.

47 Olde KP, Delemarre JF, Alons $\mathrm{CL}$, et al. The prognostic significance of morphometry for squamous cell hyperplasia of the laryngeal epithelium. Acto Otolaryngol 1986; 102:124-30. 